\title{
One-Dimensional Array of Au Nanoparticles Fixed on Nanofibers of Organogelators by the Langmuir-Blodgett Method
}

Ryo Tsunashima, Shin-ichiro Noro, Tomoyuki Akutagawa, Takayoshi Nakamura, Tomohiro Karasawa, Hiroko Kawakami, Kazunori Toma

\section{Preparation of 1 and 2}
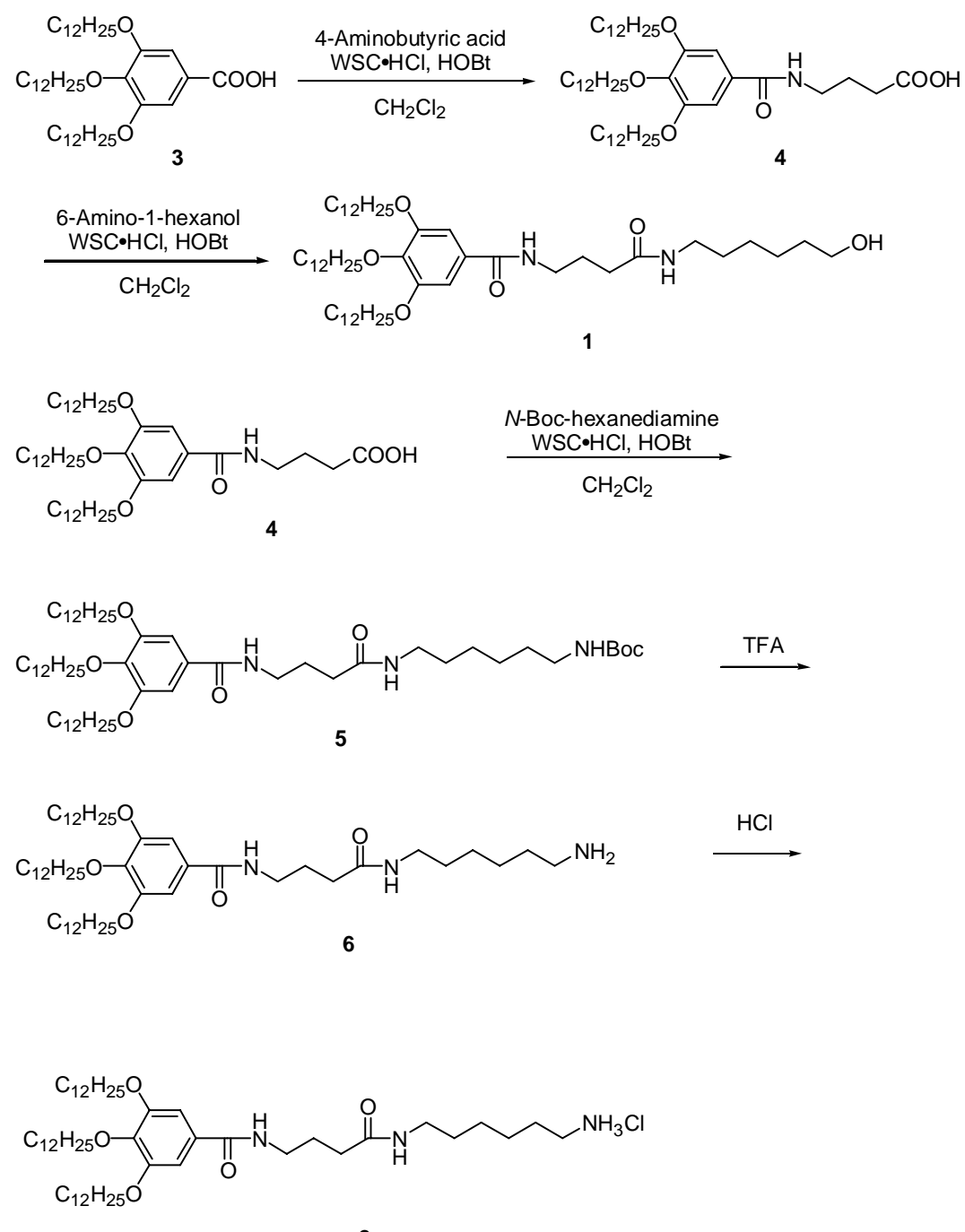

2

All reagents and solvents were purchased from commercial suppliers and were used as provided. ${ }^{1} \mathrm{H}$ NMR spectra were determined on an ECA-600 or ECP-400 spectrometer (JEOL, Tokyo, Japan). 


\section{$N$-[3,4,5-Tris(dodecyloxy)benzoyl]-4-aminobutyric acid (4).}

3,4,5-Tris(dodecyloxy)benzoic acid (3) (2.00 g, $2.97 \mathrm{mmol}$ ), water soluble carbodiimide $\mathrm{HCl}$ (742 $\mathrm{mg}, 3.87 \mathrm{mmol}$ ), and 1-hydroxybenzotriazole hydrate (595 mg, $3.89 \mathrm{mmol}$ ) were dissolved in $\mathrm{CH}_{2} \mathrm{Cl}_{2}$ $(60 \mathrm{ml})$. The mixture solution was stirred at room temperature for $1 \mathrm{~h}$, and then 4-aminobutyric acid $(2.46 \mathrm{~g}, 23.9 \mathrm{mmol})$ was added to the mixture solution. After stirring at room temperature for $2 \mathrm{~h}$, the reaction mixture was diluted with $\mathrm{CH}_{2} \mathrm{Cl}_{2}$, washed with saturated aqueous $\mathrm{NaHCO}_{3}$. The organic layer was dried over $\mathrm{MgSO}_{4}$, and concentrated in vacuo. The resulting residue was recrystallized from $\mathrm{MeOH}$ to give $4(1.64 \mathrm{~g}, 2.16 \mathrm{mmol}, 73 \%)$. ${ }^{1} \mathrm{H} \mathrm{NMR}\left(\mathrm{CDCl}_{3}\right) \delta 0.88(9 \mathrm{H}, \mathrm{t}, J=6.7 \mathrm{~Hz})$, 1.20-1.50 (54H, m), $1.75(6 \mathrm{H}, \mathrm{m}), 1.94(2 \mathrm{H}, \mathrm{m}), 2.46(2 \mathrm{H}, \mathrm{m}), 3.50(2 \mathrm{H}, \mathrm{m}), 3.98(6 \mathrm{H}, \mathrm{m}), 6.05(1 \mathrm{H}$, brs), $6.96(2 \mathrm{H}, \mathrm{s})$.

\section{$N$-[N-(6-hydroxyhexyl)-3-carbamoylpropyl]-3,4,5-tris(dodecyloxy)benzamide (1)}

4 (501 mg, $0.659 \mathrm{mmol})$, water soluble carbodiimide $\mathrm{HCl}(165 \mathrm{mg}, 0.858 \mathrm{mmol}$ ), and 1-hydroxybenzotriazole hydrate $(132 \mathrm{mg}, 0.860 \mathrm{mmol})$ were dissolved in $\mathrm{CH}_{2} \mathrm{Cl}_{2}$ (30 ml). The mixture solution was stirred at room temperature for $1 \mathrm{~h}$, and then 6-amino-1-hexanol (159 g, 1.36 mmol) was added to the mixture solution. After stirring at room temperature for $3 \mathrm{~h}$, the reaction mixture was diluted with $\mathrm{CH}_{2} \mathrm{Cl}_{2}$, washed with saturated aqueous $\mathrm{NaHCO}_{3}$. The organic layer was dried over $\mathrm{MgSO}_{4}$, and concentrated in vacuo. The resulting residue was recrystallized from $\mathrm{MeOH}$ to give 1 (427 mg, $0.497 \mathrm{mmol}, 75 \%) .{ }^{1} \mathrm{H} \mathrm{NMR}\left(\mathrm{CDCl}_{3}\right) \delta 0.88(9 \mathrm{H}, \mathrm{t}, J=6.9 \mathrm{~Hz}), 1.25-1.38(52 \mathrm{H}$, m), $1.46(8 \mathrm{H}, \mathrm{m}), 1.52(2 \mathrm{H}, \mathrm{q}, J=6.9 \mathrm{~Hz}), 1.66,(2 \mathrm{H}, \mathrm{m}), 1.73(2 \mathrm{H}, \mathrm{m}), 1.82(4 \mathrm{H}, \mathrm{m}), 1.96(2 \mathrm{H}, \mathrm{m})$, $2.31(2 \mathrm{H}, \mathrm{t}, J=6.2 \mathrm{~Hz}), 3.22(2 \mathrm{H}, \mathrm{q}, J=6.2 \mathrm{~Hz}), 3.49(2 \mathrm{H}, \mathrm{dt}, J=6.2,5.5 \mathrm{~Hz}), 3.61(2 \mathrm{H}, \mathrm{dt}, J=6.2$, $5.5 \mathrm{~Hz}), 3.98(2 \mathrm{H}, \mathrm{t}, J=6.6 \mathrm{~Hz}), 4.03(4 \mathrm{H}, \mathrm{t}, J=6.6 \mathrm{~Hz}), 6.03(1 \mathrm{H}, \mathrm{t}, J=5.5 \mathrm{~Hz}), 7.04(2 \mathrm{H}, \mathrm{s}), 7.11$ $(1 \mathrm{H}, \mathrm{t}, J=5.5 \mathrm{~Hz})$.

\section{$N$-[N-(N-Boc-6-aminohexyl)-3-carbamoylpropyl]-3,4,5-tris(dodecyloxy)benzamide (5).}

4 (502 mg, $0.660 \mathrm{mmol})$, water soluble carbodiimide $\mathrm{HCl}(166 \mathrm{mg}, 0.866 \mathrm{mmol})$, and 1-hydroxybenzotriazole hydrate $(133 \mathrm{mg}, 0.869 \mathrm{mmol})$ were dissolved in $\mathrm{CH}_{2} \mathrm{Cl}_{2}(30 \mathrm{ml})$. The mixture solution was stirred at room temperature for $1 \mathrm{~h}$, and then N-Boc-hexanediamine $(0.29 \mathrm{ml}$, $1.32 \mathrm{mmol}$ ) was added to the mixture solution. After stirring at room temperature for $2.5 \mathrm{~h}$, the reaction mixture was diluted with $\mathrm{CH}_{2} \mathrm{Cl}_{2}$, washed with saturated aqueous $\mathrm{NaHCO}_{3}$. The organic layer was dried over $\mathrm{MgSO}_{4}$, and concentrated in vacuo. The resulting residue was purified by silica

gel column chromatography $\left(\mathrm{CHCl}_{3} / \mathrm{MeOH} 19: 1\right)$ to give 5 (579 mg, $\left.0.594 \mathrm{mmol}, 90 \%\right)$. ${ }^{1} \mathrm{H}$ NMR $\left(\mathrm{CDCl}_{3}\right) \delta 0.88(9 \mathrm{H}, \mathrm{t}, J=6.8 \mathrm{~Hz}), 1.22-1.38(52 \mathrm{H}, \mathrm{m}), 1.43(9 \mathrm{H}, \mathrm{s}), 1.49(10 \mathrm{H}, \mathrm{m}), 1.74,(2 \mathrm{H}, \mathrm{m})$, $1.84(4 \mathrm{H}$, quint, $J=6.6 \mathrm{~Hz}), 1.95(2 \mathrm{H}$, quint, $J=6.3 \mathrm{~Hz}), 2.33(2 \mathrm{H}, \mathrm{t}, J=6.3 \mathrm{~Hz}), 3.01(2 \mathrm{H}, \mathrm{q}, J=6.3$ 
$\mathrm{Hz}), 3.21(2 \mathrm{H}, \mathrm{q}, J=6.3 \mathrm{~Hz}), 3.48(2 \mathrm{H}, \mathrm{q}, J=6.3 \mathrm{~Hz}), 3.98(2 \mathrm{H}, \mathrm{t}, J=6.6 \mathrm{~Hz}), 4.02(4 \mathrm{H}, \mathrm{t}, J=6.6$ $\mathrm{Hz}), 4.60(1 \mathrm{H}, \mathrm{brs}), 6.03(1 \mathrm{H}, \mathrm{t}, J=5.5 \mathrm{~Hz}), 6.20(1 \mathrm{H}, \mathrm{brs}), 7.05(2 \mathrm{H}, \mathrm{s})$.

\section{$N$-[N-(6-Aminohexyl)-3-carbamoylpropyl]-3,4,5-tris(dodecyloxy)benzamide (6).}

A solution of $5(101 \mathrm{mg}, 0.106 \mathrm{mmol})$ in trifluoroacetic acid $(1.5 \mathrm{ml})$ was stirred at room temperature for $3 \mathrm{~h}$, and the reaction mixture was concentrated in vacuo. The resulting residue was recrystallized from $\mathrm{MeOH}$ to give $6(52.3 \mathrm{mg}, 0.0609 \mathrm{mmol}, 58 \%) .{ }^{1} \mathrm{H} \mathrm{NMR}\left(\mathrm{CDCl}_{3}\right) \delta 0.88(9 \mathrm{H}, \mathrm{t}, J$ $=6.8 \mathrm{~Hz}), 1.21-1.38(52 \mathrm{H}, \mathrm{m}), 1.44(8 \mathrm{H}, \mathrm{m}), 1.63(2 \mathrm{H}, \mathrm{m}), 1.71-1.85(6 \mathrm{H}, \mathrm{m}), 1.87(2 \mathrm{H}, \mathrm{m}), 2.26$ $(2 \mathrm{H}, \mathrm{t}, J=6.3 \mathrm{~Hz}), 2.92(2 \mathrm{H}, \mathrm{brs}), 3.16(2 \mathrm{H}, \mathrm{m}), 3.40(2 \mathrm{H}, \mathrm{m}), 3.97(6 \mathrm{H}, \mathrm{t}, J=6.5 \mathrm{~Hz}), 7.05(2 \mathrm{H}, \mathrm{s})$, 7.51 (1H, brs), 8.15 (2H, brs).

\section{$N$-[N-(6-aminohexyl)-3-carbamoylpropyl]-3,4,5-tris(dodecyloxy)benzamide hydrochloride (2)}

A solution of $6(23 \mathrm{mg}, 0.027 \mathrm{mmol})$ in $\mathrm{MeOH}(4.0 \mathrm{ml})$ and $\mathrm{HCl}(0.05 \mathrm{mmol})$ was stirred at room temperature for $10 \mathrm{~min}$, and the reaction mixture was concentrated in vacuo. The resulting residue was recrystallized from $\mathrm{CHCl}_{3}$ to give $2(15 \mathrm{mg}, 0.016 \mathrm{mmol}, 59 \%) .{ }^{1} \mathrm{H} \mathrm{NMR}\left(\mathrm{CDCl}_{3}\right) \delta 0.87(9 \mathrm{H}$, t, $J=6.6 \mathrm{~Hz}), 1.21-1.36(52 \mathrm{H}, \mathrm{m}), 1.44(8 \mathrm{H}, \mathrm{m}), 1.69-1.81(8 \mathrm{H}, \mathrm{m}), 1.91(2 \mathrm{H}, \mathrm{m}), 2.34(2 \mathrm{H}, \mathrm{m}), 2.98$ $(2 \mathrm{H}$, brs), $3.18(2 \mathrm{H}, \mathrm{m}), 3.41(2 \mathrm{H}, \mathrm{m}), 3.98(6 \mathrm{H}, \mathrm{m}), 7.11(2 \mathrm{H}, \mathrm{s}), 7.70$ (1H, brs), 8.31 (3H, brs).

\section{AFM images of films}


Figure S1. AFM image of LB film of 1 on 3-aminopropyl-trimethoxysilan coated silicon substrate.

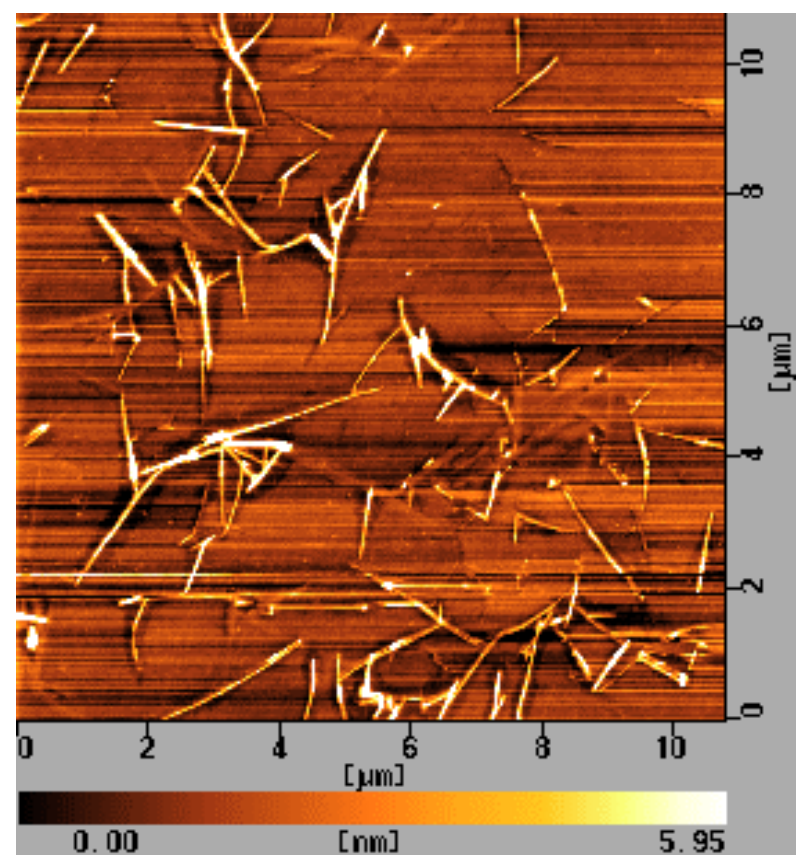

Figure S2. AFM image of cast film of 1

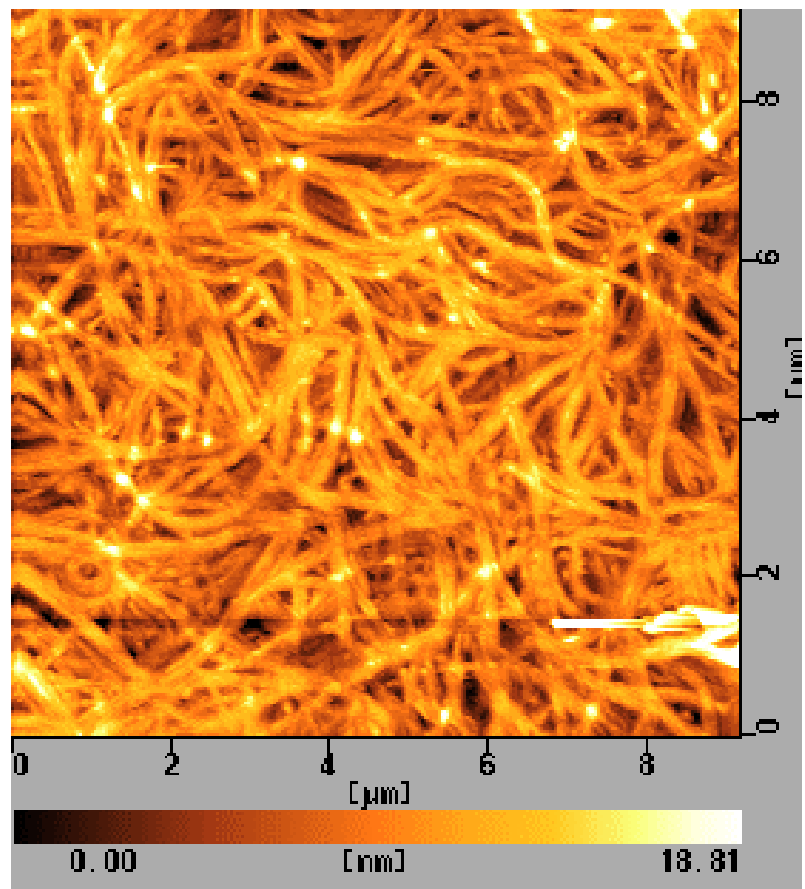

Figure S3. AFM image of a mixed LB film (mixing ratio 1:2 $=60: 40$ ) deposited at $35 \mathrm{mN} / \mathrm{m}$. 


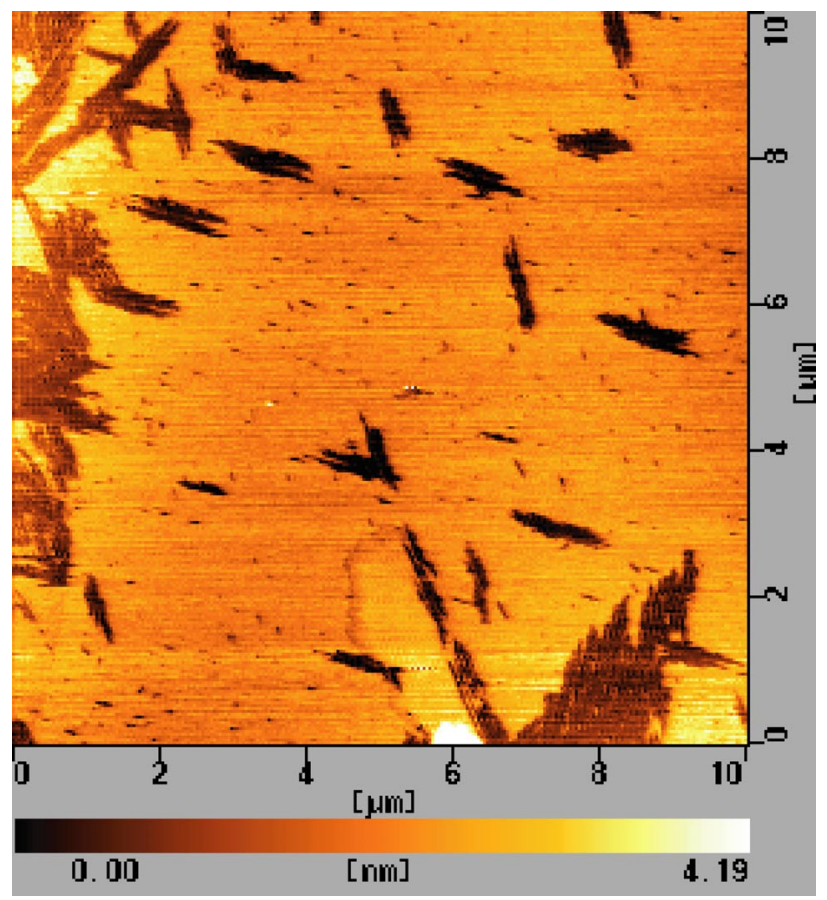

Figure S4. Topographic image (left) and LM-FFM image (right) of a LB film of $\mathbf{1}$ transferred at 25 $\mathrm{mN} / \mathrm{m}$. Topographic image showed similar heights for the fiber and uniaxial molecular assembly, while LM-FFM images showed clear difference between fiber (indicated by arrow in LM-FFM image) and the uniaxial molecular assembly because of the difference in surface oriented groups.
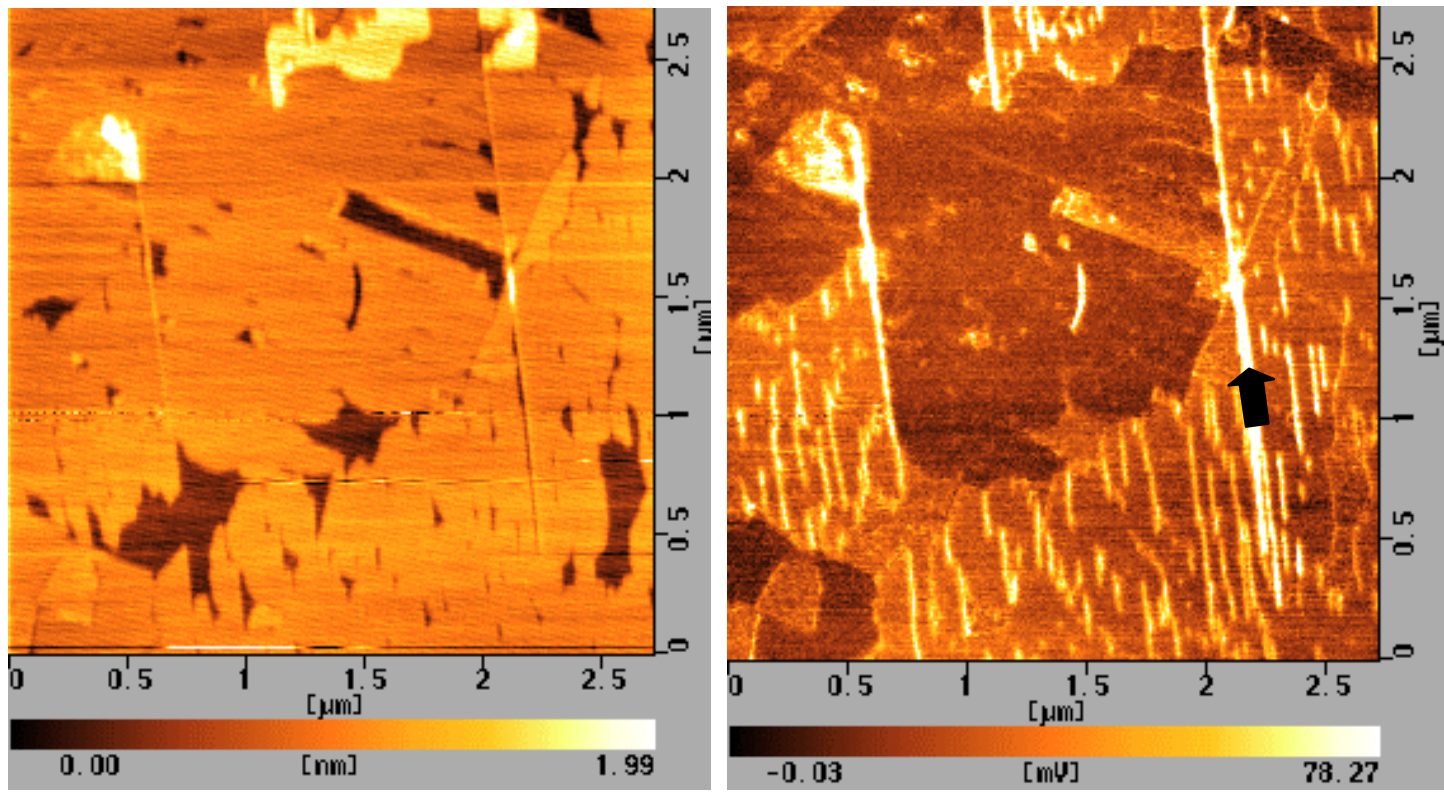

Figure S5. Topographic image (left) and LM-FFM image (right) of mixed LB film (1:2= 80:20) deposited at $35 \mathrm{mN} / \mathrm{m}$. In the AFM and LM-FFM images, fibrous structures and uniaxially oriented 
molecular assembly were observed. The anisotropic properties of their frictional signals were similar to those of $\mathbf{1}$. The fibrous structure (indicated by white circle; $\theta=95^{\circ}$ ) had higher frictional signals than others.
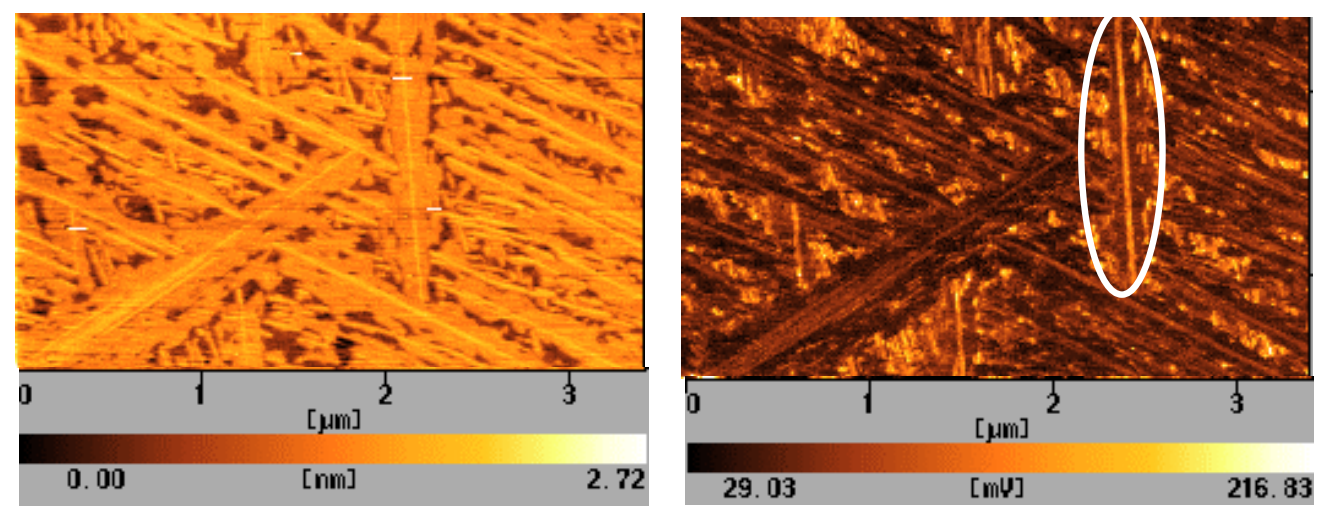

Figure S6. AFM image of $\mathbf{1}$ deposited at $35 \mathrm{mN} / \mathrm{m}$, after casting Au nanoparticles on LB film.

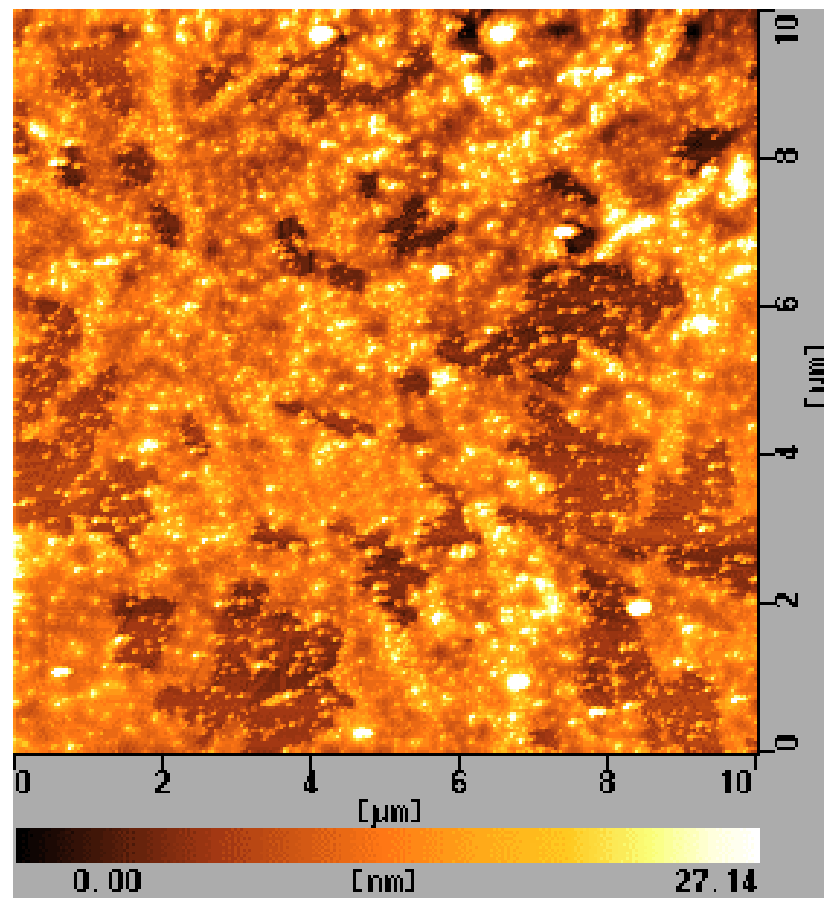

